\title{
Design and Implementation of a Wireless Networked Water Level Control System
}

\author{
Lixuan Lu1, Ross Lewis², Ming Hu², Ryan Lin² \\ ${ }^{1}$ Faculty of Energy Systems and Nuclear Science, Faculty of Engineering and Applied Science, University of \\ Ontario Institute of Technology, Oshawa, Canada \\ ${ }^{2}$ Faculty of Energy Systems and Nuclear Science, University of Ontario Institute of Technology, Oshawa, Canada \\ Email: Lixuan.Lu@uoit.ca
}

Received February 2015

\begin{abstract}
The design of a wireless water level control system is introduced and discussed in detail. In this system, the wireless Proportional Integral (PI) controller is developed using the LabVIEW graphical user programming language. Zigbee wireless technology is chosen for the wireless data transfer system. The experimental testbed was built and the system software and hardware were implemented. In order to compare the performance of the wired and wireless system, a corresponding wired water level control system was built. Experimental results show that under the same PI parameters, the settling time of the wired system is 3.3 times faster than the wireless system. However, the percent overshoot using the wireless controller is $4 \%$ smaller.
\end{abstract}

\section{Keywords}

Wireless Networked Control System, Zigbee Wireless Protocol, Labview Graphical Programming

\section{Introduction}

Traditionally, control systems rely on physical connections (wires and cables) to interconnect all the components within the system, such as sensors and actuators. Recently, with the advancements made in wireless technology, and with systems increasing in reliability, it is becoming more and more feasible to consider the application of wireless process control systems to large scale industrial applications [1]-[3].

Some of the anticipated benefits of an eventual wireless implementation include: (1) reduction of costs, since the need for long wires will be eliminated; (2) provision of control system redundancy, since the wireless system could act as a backup in the event of a loss of primary (wired) system control; and (3) simplification of maintenance, since maintenance will be localized to a few wireless nodes versus hundreds of feet of cables.

The objective of this research is to design and implement a wired and a wireless networked water level control system, so that the performance of these two systems can be compared. These implementations are carried out in a laboratory environment. The purpose is to show that wireless technology could produce suitable, sustainable and comparable results with the control algorithm implemented.

The paper is organized as follows: Firstly, the overall design of the wired and wireless system is presented.

How to cite this paper: Lu, L.X., Lewis, R., Hu, M. and Lin, R. (2015) Design and Implementation of a Wireless Networked Water Level Control System. Journal of Computer and Communications, 3, 159-163.

http://dx.doi.org/10.4236/jcc.2015.35020 
This includes the overall system structure and Zigbee wireless communication design. Subsequently, the physical system is introduced with specific selection of individual components. Wired and wireless controllers are presented. Following this, the performance results of both the wired and wireless water level system are compared. These include the transient responses and setting times of the two systems. Finally, conclusions are drawn.

\section{System Design}

\subsection{Functional and Performance Requirements}

In order to show that wireless control system could be a feasible idea to control water level in a closed loop system, both functional and performance requirements described below are considered.

There are four functional requirements that have to be met in order for the system to work: 1) Be able to control inflow; 2) Be able to control outflow; 3) Be able to measure the water level; and 4) Be able to communicate data in real time. Meanwhile, three performance requirements have to be met: 1) All equipment should operate with consistency under the same circumstances; 2) The wired system should produce consistent results with the same input; and 3) The wireless system should produce consistent results with the same input.

\subsection{Wired System Design}

Based on the functional and performance requirements, the overall design of the wired system is shown in Figure 1. In this design, the proportional valve is used at the inflow of the process tank to control the overall inflow rate. The open tank is raised above the process tank. By using gravity and the pressure height difference, the flow before the proportional valve is then provided. The pump is used to control the outflow. It is operated under DC power and can be operated proportionally in accordance to the supplied power. An ultrasonic level sensor is used to provide the level measurement. Signals from the proportional valve and the level sensor are sent to a PI controller, which is implemented in Lab VIEW. The controller then sends commands to the proportional valve to control the water level to a specified set point.

\subsection{Wireless System Design}

For the wireless system, the wires between the proportional valve to the controller and the level sensor to the controller are eliminated. Instead, these signals are transmitted wirelessly. Since the wireless system use wireless protocols in place of cables to transmit data, it is important to understand the various protocols available. Three most prominent wireless protocols are considered in this research: Wi-Fi (IEEE 802.11), Bluetooth and ZigBee (IEEE 802.15.4) [4]-[6]. Zigbee protocol is chosen for this project. ZigBee is mainly designed for measurement and control. It operates in the $2.4 \mathrm{GHz}$ band and is able to connect up to 256 devices per network. It has a signal radius of $30 \mathrm{~m}$, and can accommodate three different topologies: star, peer-to-peer and mesh [7]. ZigBee also

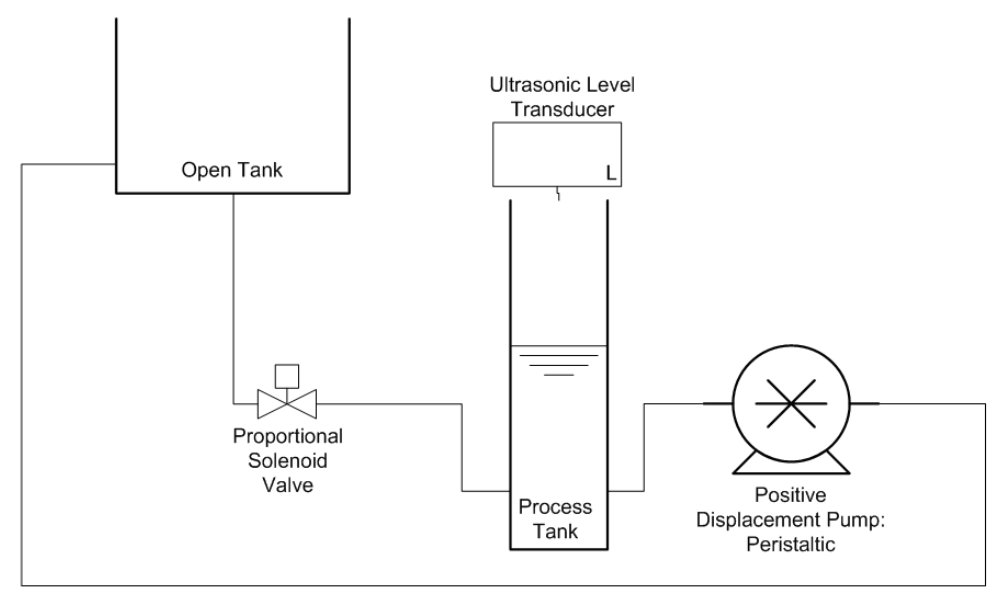

Figure 1. Wired water level control system design. 
uses CSMA/CA and requires a mandatory RTS/CTS handshake before the data will be sent. When the channel is not busy, the data will be sent immediately.

\section{System Implementation-Wired System}

After comparison of the advantages and disadvantages of different types of devices, the components chosen to build the wired system are as follows: 1) peristaltic pump; 2) paddlewheel flow meter; 3) ultrasonic level sensor; 4) general service and proportional solenoid valves; 5) nylon tubing and 6) $2 \mathrm{~L}$ transparent graduated cylinder. Figure 2 is the overall system with the actual equipment used.

A PI controller is designed in LabVIEW to perform control of liquid level. The control parameters are tuned according to Zigler-Nicoles method [8]. The block diagram of the controller for the wired system is shown in Figure 3.

\section{System Implementation-Wireless System}

Several potential configurations for wireless communication have been proposed and the final design is shown in Figure 4. The programmable logic controller from Automation direct cooperation can be programmed and managed by its design software called Direct SOFT 5. With this software, the functions of converting analog values to digital values and combining multiple data into a single data string can be achieved. The Zigbee wireless modem performance was tested by software X-CTU which was provided with the Zigbee package [9]. The

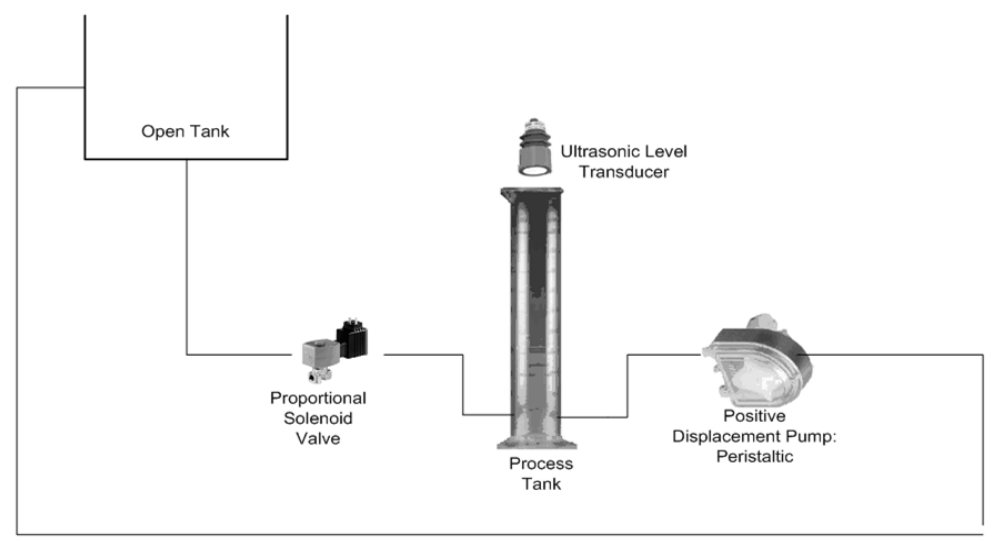

Figure 2. Wired level control system implementation.

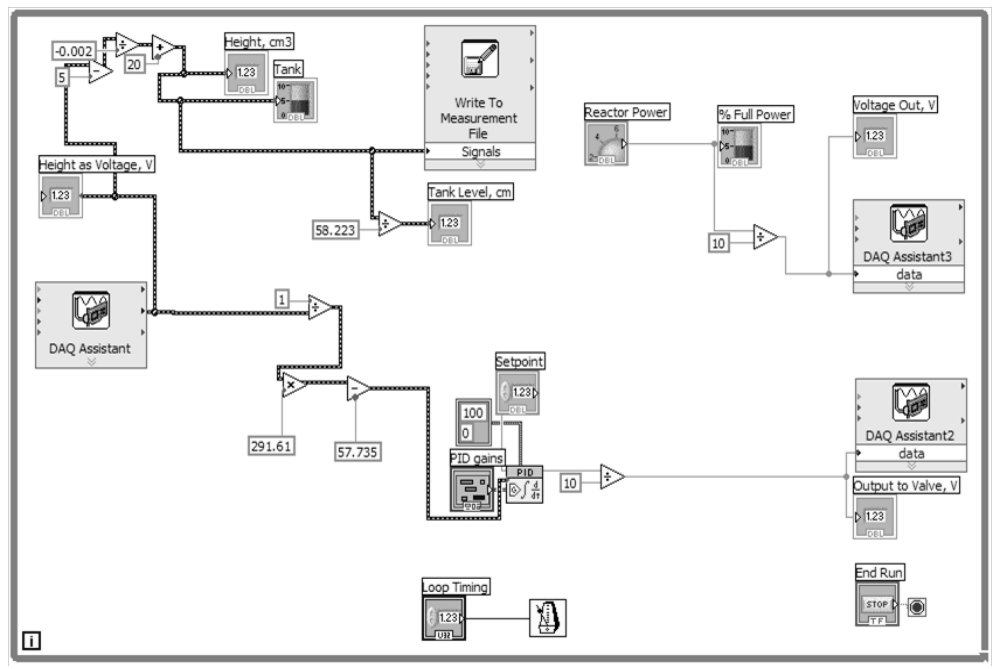

Figure 3. Block diagram of the wired PI controller. 
method was to create a wireless data transfer loop, then calculate the data transfer time out and package lost. The wireless controller is also implemented in LabVIEW. The block diagram of the wireless controller is shown in Figure 5.

\section{Experimental Results}

Six experiments were run with two sets of different PI parameters for both the wired and wireless systems. These experiments show comparable results. The comparison of the responses of these two systems is shown in Figure 6. As can be seen, for $\mathrm{Kp}=4$ and $\mathrm{Ki}=0.020$, the wireless system was found to be less stable than the wired system. There was more noise and rapid degeneration of the signal during the wireless runs, which resulted in longer settling times. Interestingly enough, the wireless system offered better performance in terms of the percent overshoot, being marginally (4\%) less than the wired system's percent overshoot. In general, the

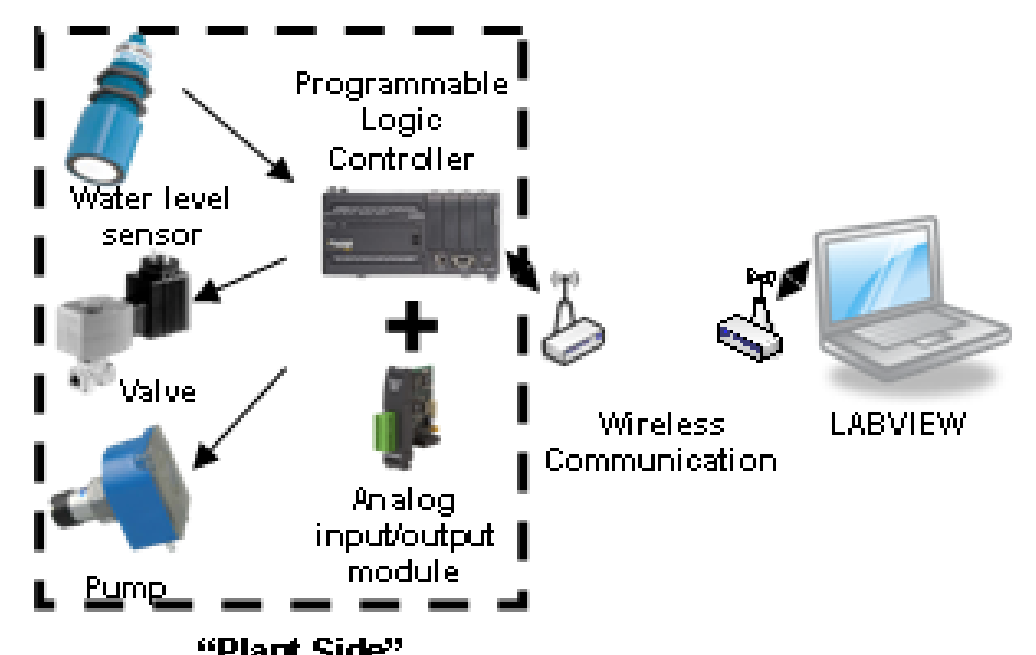

Figure 4. Wireless communication layout.

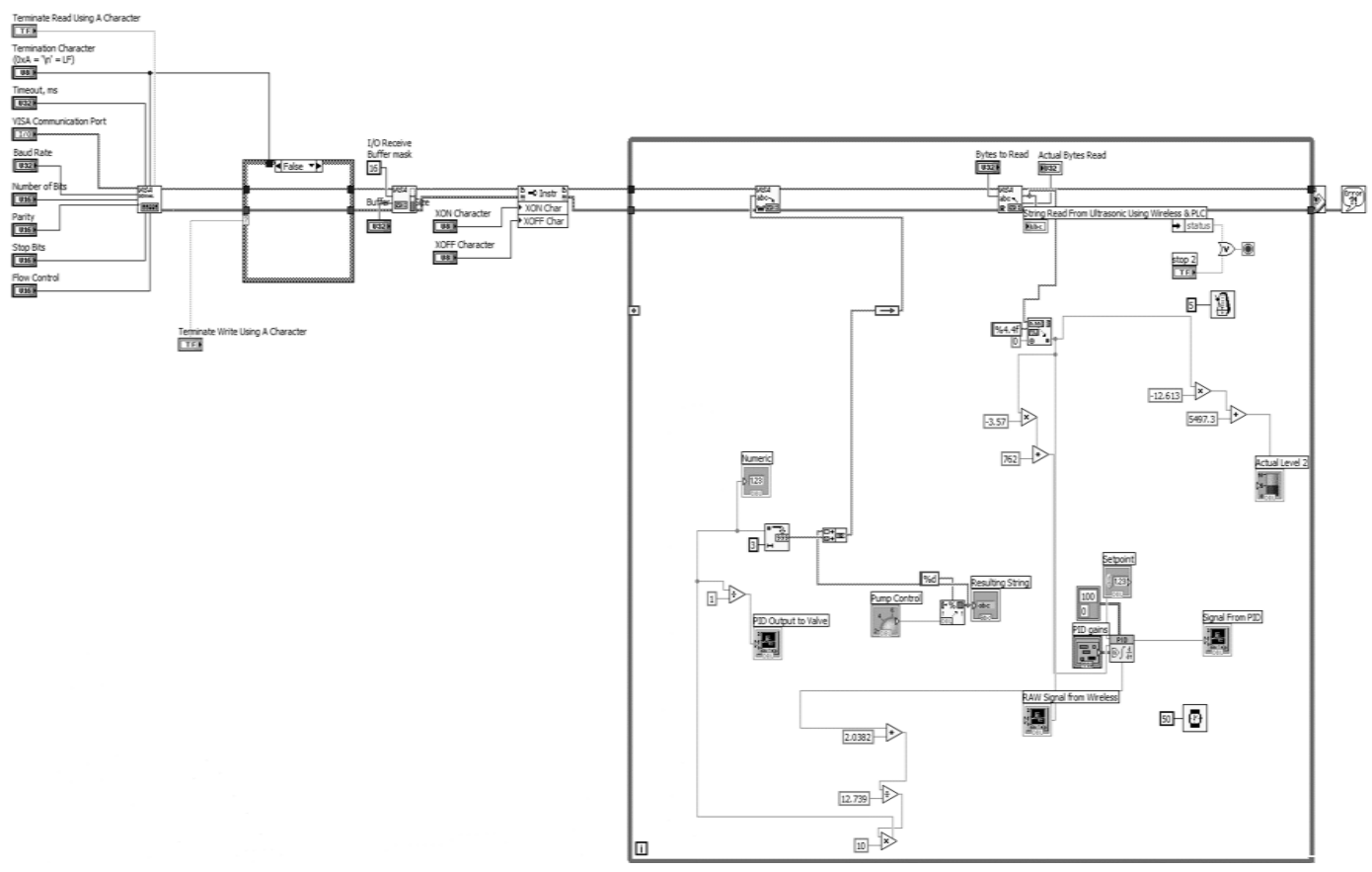

Figure 5. Block diagram of the wireless PI controller. 


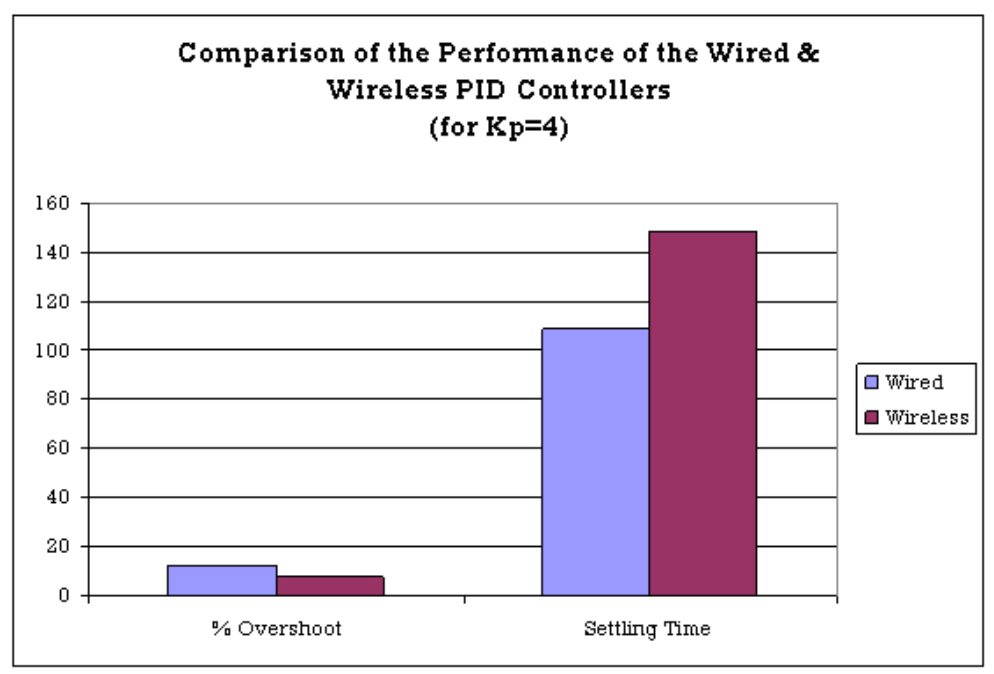

Figure 6. Summary of the performance data for wired vs. wireless @ Kp =4, $\mathrm{Ki}=0.020$.

wired system offered lower settling times. However, the percent overshoot exceeded that of the wireless system by a factor of 3.3.

\section{Conclusion}

In conclusion, a wireless water level control system is designed and implemented. Prior to implementing the wireless system, the wired system is constructed and its performance tested. The design are carried out based on both functional and performance requirements. Different components and equipment are compared and the optimal ones selected to construct the physical system. Both wired and wireless controllers are implemented in LabVIEW. Communication through Zigbee wireless channel is achieved. The performance of the wired and wireless system is compared with different control parameters. It is found that the wired system revealed faster settling time. However the percent overshoot using the wireless controller was found to be smaller.

\section{References}

[1] Katayama, T., Mckelvey, T., Sano, A., Cassandras, C. and Camp, M. (2005) Trends in Systems and Signals. Proc. 16th IFAC World Congress.

[2] Hossain, A. and Fathizadeh, M. (2004) Wireless Interface to New and Retrofitting of Existing Industrial Systems for Monitoring, Controlling and Data Acquisition in a Noisy Industrial Environment. Proc. of the ISA/IEEE Sensors for Industry Conference, 139-145.

[3] Naman, A.T., Abdulmuin, M.Z. and Arof, H. (2000) Implementation and Performance Evaluation of a Wireless Feedback Loop for Water Level Control. Proc. of TENCON 2, 56-59. http://dx.doi.org/10.1109/TENCON.2000.888388

[4] Geier, J. (2002) 802.11 MAC Layer Defined.

[5] Bluetooth Website, accessed on Feb. 11, 2015. www.bluetooth.com

[6] Ye, W. (2005) Medium Access Control in Wireless Sensor Networks. Guest Lecture for University of California, Los Angeles.

[7] ZigBee Tutorial, accessed on Feb. 11, 2015. http://www.tutorial-reports.com/wireless/zigbee/tutorial.php

[8] Dorf, R.C. and Bishop, R.H. (2005) Modern Control Systems. Pearson Prentice Hall, Upper Saddle River.

[9] XBee-PRO PKG-U USB RF Modem. MaxStream, Inc., Lindon, 2007. 